\title{
Sparse cryo-STEM tomography for biological samples
}

\author{
Antoine Cossa ${ }^{1}$, Véronique Arluison ${ }^{2}$ and Sylvain Trépout ${ }^{3}$
}

${ }^{1}$ Université Paris-Saclay, Orsay, France, Ile-de-France, France, ${ }^{2}$ Laboratoire Léon Brillouin LLB, CEA, CNRS UMR12, Université Paris Saclay, CEA Saclay, Gif-sur-Yvette, France, Ile-de-France, France, ${ }^{3}$ Institut Curie, Université PSL, CNRS UMS2016, INSERM US43, Multimodal Imaging Center, 91400 Orsay, France, Ile-deFrance, France

Cryo-electron tomography (Cryo-ET) enables to recover 3D information of cryo-fixed samples in a near-native state [Lučić et al. 2013] with a thickness up to $250 \mathrm{~nm}$ [Aoyama et al. 2008]. Thicker samples (up to $1 \mu \mathrm{m}$ ) can be studied in scanning transmission electron microscopy (STEM), which consists in the raster scanning of a (sub-)nanometric convergent electron beam focused at the sample plane [Midgley $\&$ Weyland2003][Pennycook 2012]. At equivalent electron dose, STEM induces less radiation damage to the sample than classical transmission electron microscopy (TEM) [Wolf et al. 2014] and is able to image micrometer thick cryo-fixed biological samples in 3D (see Fig. 1) [Trépout 2020]. However, in order to image thicker specimens or to reach higher magnifications we need to increase the dwell time or to sample the specimen more densely, respectively. This will inevitably increase the electron dose and the beam damage proportionally. The need to further reduce the electron dose emerges.

In this work, we present a sparse cryo-STET acquisition scheme. Instead of collecting a full frame for each tilt-angle, we collect only a fraction of the pixels. This permits to dramatically reduce the radiation dose received by the sample on each frame and across the full tilt-series. Multiple sparse data collection schemes have been previously investigated at our lab [Trépout 2019] (see Fig. 2). Before aligning the tilt-series, a preprocessing step is necessary to recover the missing information due to the data sparsity. Multiple inpainting methods are commonly used such as discrete cosine transform (DCT) [Garcia 2009], and shearlets transform [Kutynioket al. 2014]. Then the 3D volume can be reconstructed using classical methods like weighted backprojection (WBP) [Radermacher 2006] implemented in IMOD [Kremer et al. 1996] or iterative algorithms such as simultaneous iterative reconstruction technique (SIRT) [Gilbert 1972] in Tomo3D [Agullero\& Fernandez 2011].

To demonstrate the advantages of a sparse cryo-STET acquisition scheme, we applied our method on a sample that is too thick ( $>1 \mu \mathrm{m}$ ) to be studied using conventional TEM methods. 


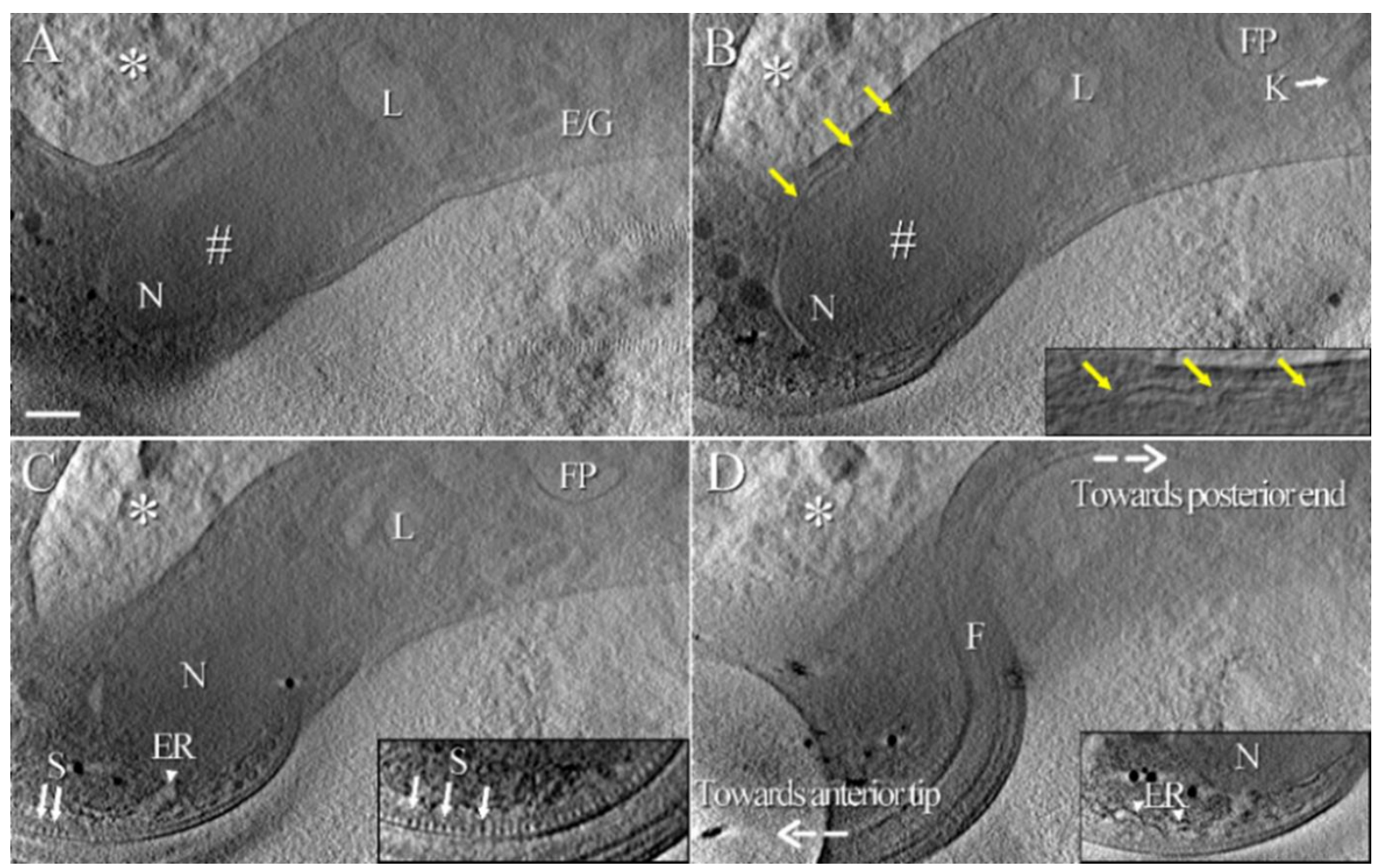

Figure 1. Figure 1. Ultrastructural organization of T. brucei observed in cryo-STET. 28 nm-thick tomographic slices showing intracellular structural elements of the cell. A) The nucleus (N), nucleolus (\#), lysosome (L) and some endosomes and/or glycosomes (E/G) are visible. B) The kinetoplast (K), flagellar pocket (FP) and location of some nuclear pore complexes (yellow arrows) are visible. C) Regularly-spaced stick-like dark densities (S, arrows) correspond to the FAZ filament located next to the FAZ-associated reticulum (ER, arrowhead). The insert is an oriented slice in which sticks (S) are visible on a larger scale. D) The flagellum (F) coils on top of the cell body. The insert is an oriented slice showing the nucleus (N) and the FAZ-associated ER. The scale bar is $300 \mathrm{~nm}$.

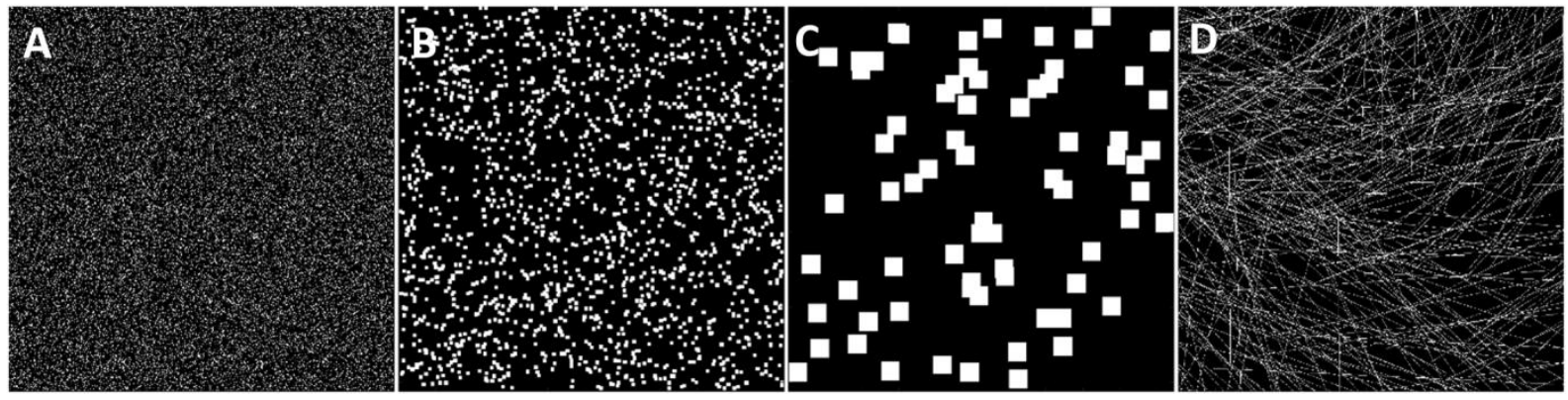

Figure 2. Figure 2. Multiples examples of sparse data collection. White pixels, representing $15 \%$ of the total number of pixels of the image, are arranged as A) individual pixels, B) $5 \times 5$ pixels patches, C) $25 \times 25$ pixels patches and D) oriented lines.

\section{References}

[1] Lučićet al., "Cryo-electron tomography: The challenge of doing structural biology in situ," J. Cell Biol., vol. 202, no. 3, pp. 407-419, 2013.

[2] Aoyama et al., "STEM tomography for thick biological specimens," Ultramicroscopy, vol. 109, no. 1, pp. 70-80, 2008. 
[3] Midgley \&Weyland, "3D electron microscopy in the physical sciences: the development of Z-contrast and EFTEM tomography," p. 19, 2003.

[4] Pennycook, "Seeing the atoms more clearly: STEM imaging from the Crewe era to today," Ultramicroscopy, vol. 123, pp. 28-37, 2012.

[5] Wolf et al., "Cryo-scanning transmission electron tomography of vitrified cells," Nat. Methods, vol. 11, no. 4, pp. 423-428, 2014.

[6] Trépout, "In situ structural analysis of the flagellum attachment zone in Trypanosoma brucei using cryo-scanning transmission electron tomography," J. Struct. Biol. X, vol. 4, p. 100033, 2020.

[7] Trépout, "Tomographic Collection of Block-Based Sparse STEM Images: Practical Implementation and Impact on the Quality of the 3D Reconstructed Volume," Materials, vol. 12, no. 14, p. 2281, 2019.

[8] Garcia, "Robust smoothing of gridded data in one and higher dimensions with missing values," Comput. Stat. Data Anal., vol. 54, no. 4, pp. 1167-1178, 2010.

[9] Kutynioket al., "Shearlets: Theory and Applications: Shearlets: Theory and Applications," GAMMMitteilungen, vol. 37, no. 2, pp. 259-280, 2014.

[10] Radermacher, "Weighted Back-projection Methods," in Electron Tomography, J. Frank, Ed. New York, NY: Springer New York, 2006, pp. 245-273.

[11] Kremer et al., "Computer Visualization of Three-Dimensional Image Data Using IMOD," J. Struct. Biol., vol. 116, no. 1, pp. 71-76, 1996.

[12] Gilbert, "Iterative methods for the three-dimensional reconstruction of an object from projections," J. Theor. Biol., vol. 36, no. 1, pp. 105-117, 1972.

[13] Agulleiro\& Fernandez, "Fast tomographic reconstruction on multicore computers," Bioinformatics, vol. 27 , no. 4, pp. 582-583, 2011. 